\title{
Using Digital Resources for Motivation and Engagement in Learning Mathematics: Reflections from Teachers and Students
}

\author{
Theodore Chao ${ }^{1}$ (D) Jason Chen ${ }^{2}$. Jon R. Star ${ }^{3}$. \\ Chris Dede ${ }^{4}$
}

Published online: 13 September 2016

C) Springer International Publishing 2016

\begin{abstract}
Students' motivation to learn mathematics often declines during the middle grades. How do we keep students engaged with learning mathematics as it gets more complex? One way is through the use of technology, such as computer games, interactive lessons, or on-line videos. Yet evidence from creating technology-based tasks and resources to motivate students to learn mathematics is mixed, partially because most interventions only loosely incorporate motivational constructs. This article is part of a larger research project examining the impact of three digital resources on students' motivation and learning in mathematics. In it, we provided resources tightly aligned to motivational constructs from research: self-efficacy, implicit theories of ability, and interest and enjoyment. Students then engaged with these resources before and after a 2-day mathematical patterns lesson. We present results from interviews and observations with eighty-eight fifth- to eighth-grade students and their ten teachers. Findings suggest that, even with a minimal encounter over 1 or 2 days, students were able to notice the motivational constructs present within these digital resources.
\end{abstract}

Keywords Implicit theories of ability $\cdot$ Middle school mathematics $\cdot$ Motivation · Self-efficacy $\cdot$ Technology $\cdot$ Video games

Theodore Chao

chao.160@osu.edu

1 College of Education and Human Ecology, The Ohio State University, 1945 N. High St, Columbus, OH 43210, USA

2 School of Education, The College of William and Mary, 301 Monticello Avenue, Williamsburg, VA 23187, USA

3 Graduate School of Education, Harvard University, 6 Appian Way, Cambridge, MA 02138, USA

4 Graduate School of Education, Harvard University, 13 Appian Way, Cambridge, MA 02138, USA 
Success in mathematics during the middle-school years (typically, ages 11 to 15 in the USA) is widely recognized as a gatekeeper of later academic and social success (Adelman 2006; Moses and Cobb 2001). During this period, student motivation to learn mathematics significantly declines (Archambault et al. 2010; Blackwell et al. 2007; Dweck 2007; Eccles-Parsons et al. 1983). According to the U.S. National Mathematics Advisory Panel report, $62 \%$ of the Algebra I teachers surveyed rated working with unmotivated students as the single most challenging aspect of teaching (Hoffer et al. 2007).

One approach to this problem of low student motivation is to use technology-based resources both to spark students' interest in learning mathematics and to develop greater confidence in mathematical problem solving. Historically, many teachers use technology in their mathematics classes in a variety of ways, ranging in complexity and cost from repurposing commercially available television programs to utilizing computer games.

Even as many teachers embrace technology in their classrooms, the evidence surrounding the effectiveness of using it to ignite student interest in the academic content is sparse (Chen et al. 2016; Chen et al. 2014; Moos and Marroquin 2010). One reason for this lack of knowledge is because technology is not often aligned with specific aspects of motivation and content. Rather, the technology is incorrectly assumed to be generally and comprehensively motivating - the presumption that children will be interested in learning or become more confident mathematically simply because of the presence of technology.

A second reason for these uncertain results is that much of the research on technologybased resources and tasks explores motivation as an afterthought rather than a central part of the design process. Both these reasons are likely due to a lack of alignment between theoretical grounding in well-studied motivation constructs and educational technology design (Chen et al. 2012; Moos and Marroquin 2010). As a result, educators and educational technology designers lack empirical evidence for which types of motivational constructs, exemplified in technology design, are useful for whom and under what types of conditions in order to enhance the learning of mathematics.

We sought to address this evidence gap by exploring how students described their experiences when working with three different kinds of digital resources - an immersive video game, an interactive website, and a commercially produced video - chosen specifically in relation to three distinct frameworks of motivation and engagement. To explore their impact on student motivation to learn mathematics, we present a qualitative analysis of the views of eighty-eight students (in grades five to eight) and their ten teachers as they participated in a week-long motivational technology intervention, during which students and teachers discussed both the specific resource they had worked with and their motivation for learning mathematics. Because the investigation was so short, we focused on the impact of the intervention on student motivation for learning mathematics, rather than trying to measure changes in mathematics content knowledge.

When we say motivation, we specifically mean students' interest in mathematics and their confidence in relation to success in mathematics (i.e., self-efficacy, implicit theories of ability, and interest and enjoyment). Two research questions guided our investigation.

(1) From the standpoint of motivation and engagement, what were students' perceptions of and experiences with the digital resources?

(2) How did teachers of mathematics perceive their students' self-efficacy, their implicit theories of ability, and their interest and enjoyment in learning mathematics? 


\section{Background Theoretical Framework}

Understanding this study requires exploring the theoretical underpinnings that went into the work with each resource. In the sub-sections below, we discuss the theories of motivation informing the chosen resources. We also examine the research on how each digital element relates to a specific motivational construct for learning: self-efficacy, implicit theories of ability, and interest and enjoyment. (See Table 1 for how each resource incorporated a specific motivational construct and aspect of mathematics learning.)

\section{Self-Efficacy and Mathematics Learning}

Mathematics is considered one of the most difficult subjects to master in school (Dweck 2000; NRC 2001). A robust belief in one's capabilities to succeed (i.e. selfefficacy) in mathematics is critical to learning mathematics. More generally, four decades of research have shown the importance of self-efficacy to outcomes such as persistence and perseverance in the face of difficulties, academic achievement, and students' choice of college majors and careers (Bandura 1997; Brown and Lent 2006; for a review, see Pajares and Urdan 2006).

Given these important outcomes tied to mathematics learning, researchers have explored factors that support self-efficacy. Bandura (1997) posited that self-efficacy is formed by people's interpretation of four main sources, two of which we target here. First, mastery experiences (the interpretations of one's past accomplishments) constitute the most powerful source of self-efficacy. Students who interpret their past mathematics experiences as successful are more likely to approach future mathematics endeavors with a strong belief in their ability to succeed. Second, observing others succeed or fail influences self-efficacy. Such vicarious experiences are especially influential when observers perceive others as being similar to themselves, which is particularly influential when individuals have little experience with the task or are uncertain about the standards by which they will be judged. For example, students who have never faced algebra before, or are uncertain about how they will be graded, might turn to siblings, friends, or narratives of others with whom they relate to as pertinent informants.

\section{Implicit Theory of Ability in Mathematics}

A related belief about competence involves students' perceptions about the nature of their mathematics abilities, their implicit theory of ability in mathematics. Dweck and

Table 1 Motivational constructs for each digital resource

\begin{tabular}{lll}
\hline Digital resource & Motivational construct & $\begin{array}{c}\text { Connected to the trains } \\
\text { lesson content? }\end{array}$ \\
\hline The game: A virtual environment & $\begin{array}{c}\text { Self-efficacy through vicarious } \\
\text { and mastery experiences }\end{array}$ & Yes \\
$\begin{array}{l}\text { Brainology: A web-based curriculum module } \\
\text { The video: an off-the-shelf PBS NOVA episode }\end{array}$ & $\begin{array}{l}\text { Interest and enjoyment } \\
\text { Implit theory of ability }\end{array}$ & $\begin{array}{l}\text { No } \\
\text { No }\end{array}$ \\
\hline
\end{tabular}


Leggett (1988) posited that individuals typically fall into one of two categories: fixed mindset or incremental/growth mindset. Students holding a fixed mindset believe that, no matter what the circumstances are, one's intellectual abilities are 'set in stone' there is little that can be done about one's 'smarts' (Blackwell et al. 2007; Cury et al. 2006; Good et al. 2012; Grant and Dweck 2003). Students holding a fixed mindset either pursue a task to demonstrate their competence or avoid one for fear of looking incompetent. These students are more likely to see effort as 'bad' and, in the face of obstacles, tend to give up prematurely and achieve only at low levels.

On the other hand, students with an incremental or growth mindset believe that, with hard work and appropriate strategies, they can increase their intellectual abilities (Dweck and Leggett 1988). Students holding an incremental mindset pursue a task or explore a situation simply for the sake of learning. Such students see effort as positive and therefore persist through tough obstacles. Ultimately, students holding an incremental mindset tend to achieve at higher levels than their peers holding a fixed mindset.

So why do students adopt one type of mindset over another? Some suggest that teachers' mindsets and methods of evaluation influence the type of mindset their students develop (Good et al. 2012; Rattan et al. 2012). Others suggest that the type of feedback a teacher provides also influences mindsets (for a review, see Dweck and Master 2009). For example, describing the outcomes and great accomplishments of individuals without emphasizing their relentless commitment can promote a fixed mindset. However, when teachers emphasize the importance of strategies and persistence, students are more inclined to view their abilities as augmentable, which can lead to an incremental mindset.

\section{Interest in and Enjoyment of Mathematics Learning}

A substantial body of literature has shown that interest in academic study declines measurably, particularly as students enter their adolescent years (which in the United States is during grades six to twelve). Many interventions, especially in mathematics, have been crafted to address this decline in academic interest. To understand the ways researchers can design interventions that target academic interest, we invoke Hidi and Renninger's (2006) four-phase model of interest development. Interests come, they claim, in two types: situational and individual. First, interests start out as situational, which are short-lived and often require substantial external support to sustain. Typically, students first experience are triggered situational interest in something which is surprising, personally relevant, or especially enjoyable. It sparks a temporary interest.

The second phase occurs when this trigger translates into a situational interest that is maintained, where students' interests are sustained through personally meaningful tasks and/or personal involvement. If such interest is maintained and developed, it can turn into an individual interest, which are more well developed and longer lasting. An emerging individual interest is typically characterized by stored knowledge, value, and positive affect, which, if sustained over time, can then develop into what is termed a well-developed individual interest, one in which students possess robust stored knowledge and value for the subject. Students at this stage are able to re-engage with tasks related to the specific subject topic or almost entirely on their own volition. 


\section{Leveraging Technology as a Motivational Tool}

The research literature includes examples of technology interventions that have successfully targeted self-efficacy, implicit theories of ability, and interest and enjoyment. Digital resources can support gains in self-efficacy when learning about science, particularly through providing mastery experiences for students, by allowing them to see the fruits of their own labor. For instance, Ketelhut et al. (2010) found that students with low initial self-efficacy who participated in a multi-user virtual environment (MUVE) called River City rated themselves more self-efficacious at inquiring scientifically. By the end of the intervention, low initial self-efficacy students behaved similarly to those with high initial self-efficacy, gathering data in the virtual world as thoroughly as students with high self-efficacy.

Similarly, Liu et al. (2006) showed that students' self-efficacy for learning science increased after participation in a computer-enhanced, problem-based learning (PBL) environment called Alien Rescue. The study found that student gains in self-efficacy came from opportunities to see themselves succeed without assistance, rather than having a teacher tell them how to succeed. These two studies illustrate how technology can support student self-efficacy by providing relevant mastery experiences - the strongest hypothesized source of self-efficacy.

Similarly, some evidence exists demonstrating the effect of digital resources targeting our second motivational construct: students' implicit theories of ability. Specifically, Dweck and colleagues have developed Brainology, ${ }^{1}$ a web-based series of learning modules designed to teach students about the incremental mindset (termed the 'growth' mindset). Dweck and colleagues have reported promising results using their paper-based growth mindset intervention (see Blackwell et al. 2007). These results showed that students in a control group, who were not taught a growth mindset, displayed a continuing downward trajectory in grades and motivation, whereas the experimental group displayed an upward one. Despite these promising findings, however, the web-based material, which is modeled on the paper-based version, lacks rigorous large-scale efficacy studies to support its claims of effectiveness.

Third, scholars have been able to show that digital resources can be deployed to recruit students' interest and enjoyment. For example, Quest Atlantis (a three-dimensional virtual world) has been shown to make particular learning goals interesting by placing them within a fun and meaningful context in which students collaboratively interact to solve problems (Barab et al. 2003). In addition, using an immersive virtual world called EcoMUVE, Chen et al. (2016) were able to show that, for students whose interest in the technology was both triggered and maintained, their individual interest in scientific inquiry was strengthened. Sparking an interest in the technology is one thing, but being able to target students' interests for the subject matter is quite another endeavor.

\section{The Three Digital Resources}

Given the theoretical framing regarding motivation discussed earlier, we sought to provide digital interventions that helped students develop their motivation (i.e., self-efficacy,

\footnotetext{
${ }^{1}$ Brainology is a registered trademark of the US company Mindset Works, Inc.
} 
implicit theories of ability, and interest and enjoyment) toward learning mathematics. (The qualitative investigation reported here is part of a larger project investigating the relationship between specific digital resources that exemplified various motivational constructs and students' motivation in mathematics, as well as their interest in pursuing STEM careers.) Our 88 fifth- to eighth-grade students were randomly assigned access to one of three digital resources centered on specific frameworks of motivation. All students participated in the same 2-day mathematics lesson on patterns.

Elsewhere, we have provided a quantitative analysis of the overall impact of the interventions on motivation (Star et al. 2014). Whereas our quantitative paper investigated the overall effects of student exposure to the digital resources, the goal of our present study was to explore how students reacted to the different resources and how connected these reflections were to the specific motivational theory frameworks imbedded within them. For example, because the first resource, a game, targeted students' self-efficacy through vicarious 'models', we wondered whether the lived experiences of students who had played the game suggested any awareness of or attention to those models.

We detail the three digital resources in Table 1.

\section{Site 1: The Game, an Immersive Virtual Environment}

The first resource involved an immersive virtual environment (henceforth, "the game"). In designing the game to bolster students' self-efficacy, we paid attention to the two sources of self-efficacy described above: mastery experiences, gained by successful interpretation of one's previous performances, and vicarious experiences, gained by observing others' actions. In the game, players are to guide a stranded space explorer on a distant planet. (See Fig. 1.)

The primary way we targeted students' self-efficacy was through mastery experiences - we presented them with incrementally more challenging tasks, akin to 'leveling up' in a commercial video game. We built in 'just-in-time' scaffolds, so that when students encountered some difficulty, the game would break the task into simpler and more achievable steps. For instance, if a student had trouble determining the number of

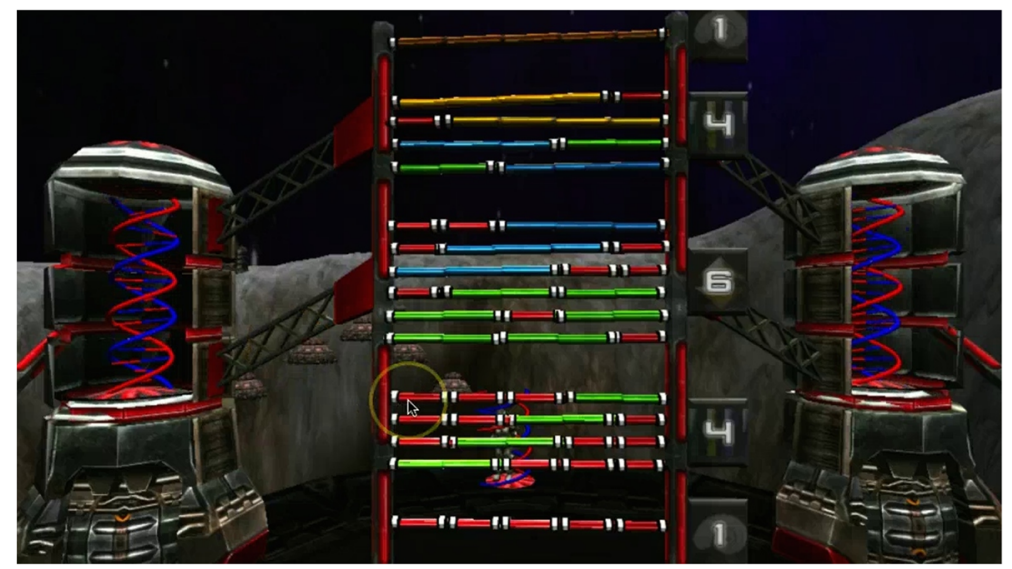

Fig. 1 A screenshot of the Immersive Virtual Environment game in which a student must solve a combinations task to free the trapped spaceship captain 
combinations of multiple-length fuses, a pop-up dialogue would suggest, "Oops, try building it out", highlighting an area in which the student could drag the different fuses to organize the various combination. (See Fig. 2.)

A secondary way in which we targeted self-efficacy was through vicarious experiences. Students watched a 5-minute video clip of real STEM professionals describing their jobs, the difficulties that they encountered with mathematics while they were in school, and the strategies they used to overcome these difficulties. By presenting students with real, fallible 'models', who were young and ethnically diverse, we hoped that students would see these individuals as relatable and would see that finding a STEM job is attainable even if, as students, they experienced significant struggles.

The game mimicked an action-oriented console video game (e.g. Playstation, Xbox) and, therefore, was the most highly interactive of the three resources. In addition, the game was the only one connected to the specific mathematical patterns task (described later). This connection of the class mathematical task to the game allowed us to see whether the game could help the students make use of the scaffolded mathematical exercises within the game - not explicitly labeled as mathematics - as a means to build their self-efficacy in solving similar pattern tasks in the classroom.

Additionally, the particular mathematical challenges in the game were directly connected to the 'trains' pattern task. Our belief was that students would be engaged in mathematical thinking while interacting with the game, based upon their exploring the various challenges, organizing the permutations of the rods within them, and then generalizing from the pattern.

\section{Site 2: Brainology ${ }^{\circledR}$, a Growth Mindset Web-Based Learning Module}

The second resource was an abridged version (created by Dweck and colleagues specifically for the present study) of the Brainology® program (www.mindsetworks.com), a web-based set of learning modules. (See Fig. 3.)

This version was tailored specifically for students in grades 5-9 and focuses on the concept that the brain is like a muscle - the harder you work it, the stronger it grows.

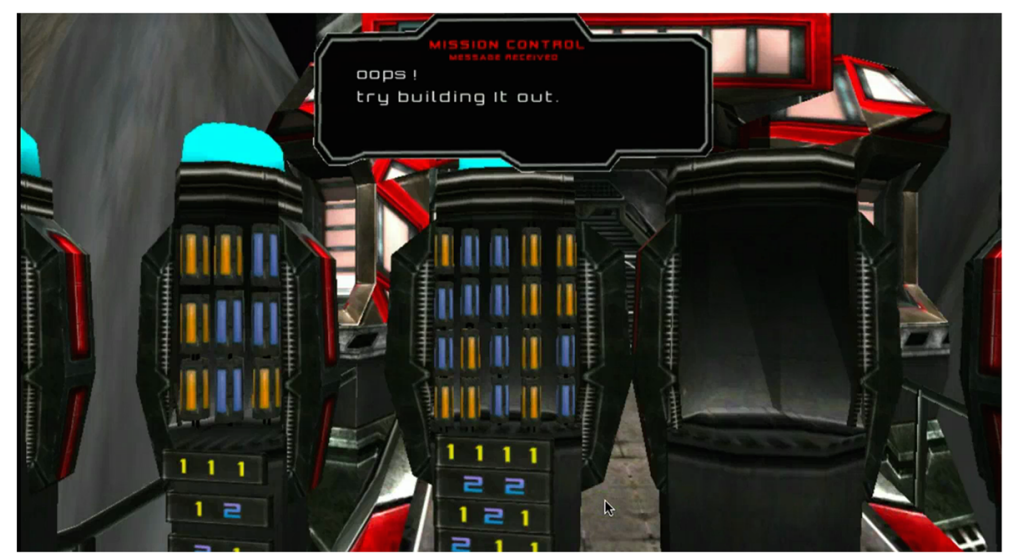

Fig. 2 A 'just in time' prompt that pops up when a student struggles with the mathematics task in the Immersive Virtual Environment game 
Through a series of tasks and games, students come to see that when they find appropriate strategies and put forth significant effort to accomplish something difficult, they can get smarter at a subject. By convincing students that the secret to success is finding good strategies, rather than having an immense intellect (a common belief in mathematics), students learn to focus on things they can control (e.g. effort, strategies), rather than on things that are out of their control (e.g. immense intellect).

Although the modules include animations and games, they still feature a linear structure and substantial text. Consequently, we felt this media type was still interesting, but not as stimulating and interactive as the game. Additionally, Brainology ${ }^{\circledR}$ was not specifically connected to any particular mathematical task or even the domain of mathematics. Dweck and her colleagues have suggested this is not a problem because targeting the belief about the nature of intelligence alone, especially in relation to a subject in which intelligence is quite salient (mathematics), results in positive outcomes. We chose this material to explore whether exposure to this belief alone could have a beneficial effect on student motivation for learning mathematics.

\section{Site 3: The Video, a Professionally Created Educational Film}

Because videos have long been used by educators to motivate and engage students in the classroom, we chose a video produced by the Public Broadcasting Service (PBS) NOVA program, Fractals: Hunting the Hidden Dimension (Schwarz and Jersey 2009). This 55-minute programme features visually appealing animations, interviews with mathematicians and accessible explanations of the mathematics of fractals and their everyday applications. (See Fig. 4.)

This video did not target a particular motivation construct, mimicking the way teachers integrate 'off-the-shelf' movies into their classrooms. Although the video was specific to the domain of mathematics, it did not focus on nor offer any particular mathematical tasks. The video generally targeted students' beliefs about the intrigue of mathematics and its relevance to everyday life - helping students see the value of

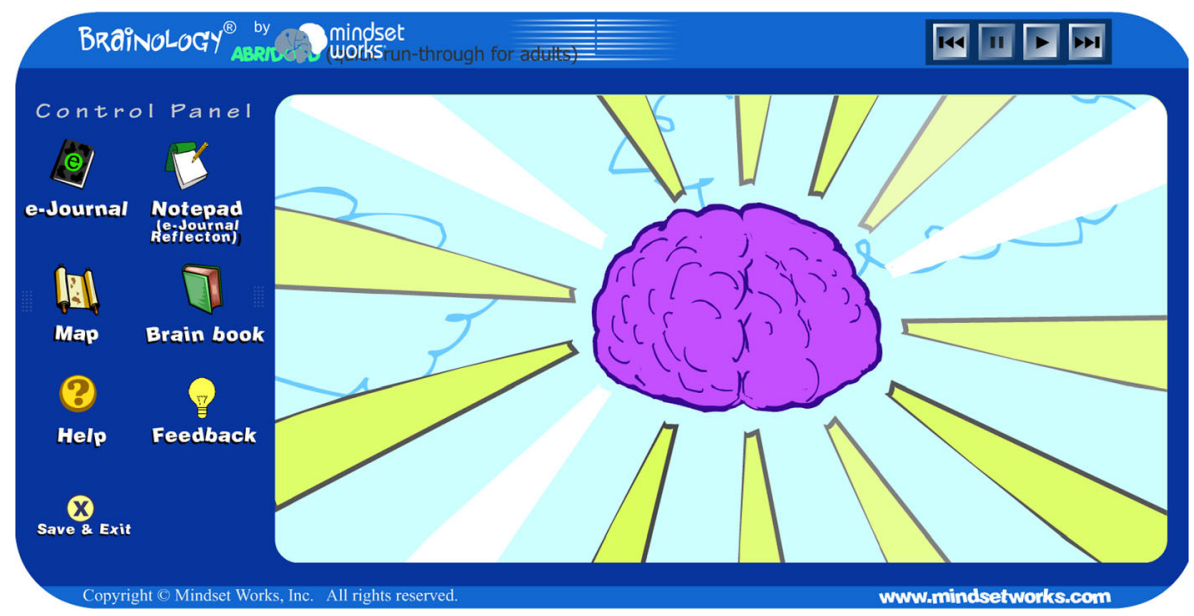

Fig. 3 A screenshot of the Brainology ${ }^{\circledR}$ module, in which virtual students guide tasks that help develop a growth mindset 


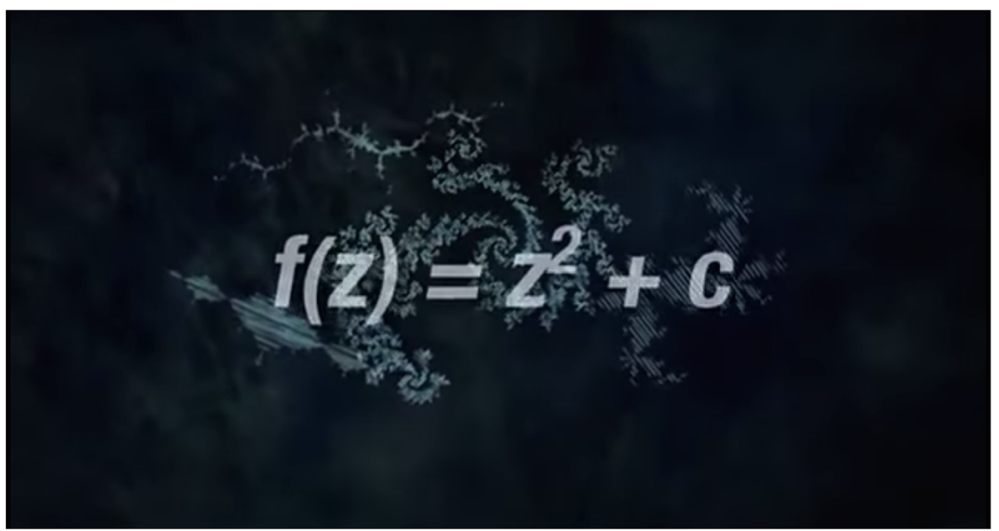

Fig. 4 A screenshot of the PBS video, which explores the mathematics behind fractals

engaging in and doing mathematics. Furthermore, the characters in the movie were presented as relatable, fallible individuals who had great enthusiasm and curiosity, which led them to find interesting applications of fractals to everyday life (e.g., special effects in movies, creating interesting patterns in tie-dye shirts). We chose this resource as a way to target students' interest in and enjoyment of mathematics without addressing their self-efficacy or implicit theories of ability.

\section{The Mathematics Lesson: A Pattern Exploration Task}

Regardless of which resource with which a student engaged, all students explored a 2day mathematical patterns lesson built around a combinatorics task (the 'trains problem'). ${ }^{2}$ This task asked students to determine the number of different integer-length 'trains' that could be created from multiple combinations of smaller integer-length 'cars'. For example, there are eight ways to create trains of length four: 1-1-1-1, 1-1-2, 1-2-1, 2-1-1, 1-3, 3-1, 2-2 and 4. (See Appendix A for the trains lesson task.)

We chose the trains problem for two reasons. First, it potentially can lead to the discovery of the Fibonacci sequence, Pascal's triangle and other important mathematical patterns. Second, the trains problem is cognitively demanding, meaning that it is useful for developing students' mathematical thinking (Stein and Lane 1996). The focus of the 2-day lesson was: How many different length-seven trains can be made? The lesson involved the collection, organization, representation, and generalization of numerical data, while requiring teachers to manage cycles of task-posing, small-group work, student sharing, and classroom discussion. The mathematical content of the trains problem was only directly connected to the game.

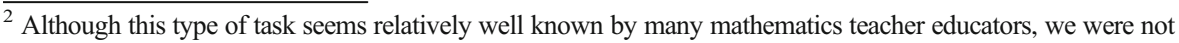
able to identify a published source that could be credited with the creation of the task in this context. We first learned about this task at the Park City Mathematics Institute in the early 2000s that was facilitated by Ben Sinwell and Bowen Kerins, neither of whom can recall the origins of the task. (I believe this task goes back at least to the mid-1950s with Caleb Gattegno's work on Cuisenaire rods. Certainly exploring related tasks drawing on the language of trains and cars is there. See Book 1 of his Mathematics with numbers in colour $-E d$.)
} 


\section{Methods}

Before the study, all teachers participated in a full day (seven hours) of professional development facilitated by one of the authors the week before implementing the 2-day lesson. It was designed to familiarize teachers with the lesson's mathematical goals and to develop the teachers' facility with implementing the trains problem. They first experienced the trains problem as their students would, and then reflected on this experience. This reflection focused on the different ways the lists of trains could be organized, how students would know they had built all trains for a given length, and why a doubling pattern emerges from this task. There was a short breakout group at the end of the day, during which teachers were introduced to their specific digital resource.

The timeline for the research was as follows (see Fig. 5). On the Monday of the week following the professional development day, the teachers introduced the particular digital resource to their students during the mathematics class, presenting the game, Brainology ${ }^{\circledR}$ or the video depending on their randomly assignment. On both Tuesday and Wednesday, teachers offered their students the trains task during their mathematics class. Researchers conducted one-on-one interviews with the teachers before the Wednesday class. On Thursday, the teachers presented a second opportunity for student involvement with their particular digital resource. On Thursday, both before and after class, researchers conducted small-group interviews with students.

The data for this study consists of student and teacher interviews from a convenience sample of 88 students in grades five through eight and their ten teachers from four elementary and middle schools in a large urban public school district in the Eastern USA. Seven of these teachers taught fifth grade, one taught seventh grade, and two taught eighth grade. The interviews were conducted both during and after the intervention, as mentioned above. Participants attended two elementary schools, which served students in kindergarten through fifth grade, and two middle schools, which served students in sixth, seventh, and eighth grade. ${ }^{3}$ We wished to observe students in both elementary schools and middle schools and these schools' geographic locations allowed our research team consistent access throughout the four-day intervention. Demographic data for the four schools can be found in Table 2.

Participating teachers in the four schools were asked to select students they felt were either highly motivated or not motivated in mathematics class. We then arranged focus groups to conduct interviews comprising from two to four students from the same class, so the students in each group had engaged with the same digital resource. The length of the 32 student interviews ranged from nine to 45 minutes. While each group had a mix of (teacher-identified) highly motivated and unmotivated students, at the time of the interviews the research team was unaware which students were which.

These audiotaped interviews were conducted in students' classrooms using a common interview protocol focusing on technology, motivation, and the specific digital resource (see Appendix B). Because the interviews were conducted during the third and the fourth day of intervention, at the time of the interview some of the students had only participated in the project for three of the four days. Sample questions from the

\footnotetext{
${ }^{3}$ The 88 students interviewed represent almost $3 \%$ of the overall number of students from these four schools who participated in the larger project and just over $0.5 \%$ of the number of students who participated in the larger research project.
} 

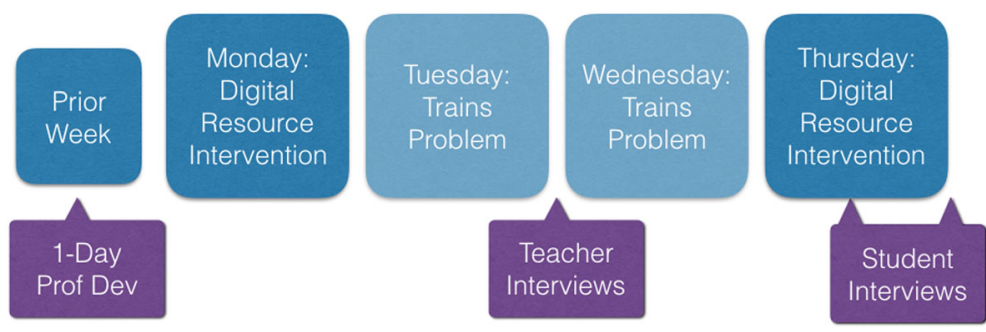

Fig. 5 A brief timeline of the research study

interview included, "Could you tell me about the technology activity that you participated in during the first day: Was it interesting? Was it fun? In what ways?"

The teacher data involved ten teachers from these four schools. ${ }^{4}$ Individual audiotaped interviews were conducted in each teacher's classrooms between the two days of the trains problem, as described above. When teachers were interviewed, they had only been involved in the intervention for two of the four days. The ten teacher interviews ranged from 25 to 65 minutes and followed a common interview protocol (see Appendix C), focusing on the teacher's background, use of technology, and reflections on the lesson. Sample questions from the interview included, "Did you think that the technology activity was something that benefitted the students? Why or why not?" Demographic data for the ten teachers can be found in Table 3.

\section{Initial Assumptions About Student and Teacher Motivation}

As mentioned above, our research questions were about both students and teachers. First, from the standpoint of motivation and engagement, we wanted to explore students' experiences with the digital resources. For this question, we hypothesized that students playing the game would report on the vicarious experiences through the STEM professional narratives and the mastery experiences from the challenges. After all, Bandura (1997) positioned these two experiences as especially powerful sources of self-efficacy. Second, we hypothesized that students working with the abridged Brainology modules would be interested in learning mathematics because implicit theories of ability have been shown to have a significant effect on students' motivation. However, we tempered those expectations because the Brainology tool has not had a substantial track record of success (see Donohoe et al. 2012).

Furthermore, this particular resource was an abridged version, focusing specifically on the growth mindset and strategies to reduce anxiety in school, which is a common feeling in mathematics (Ashcraft and Krause 2007; Ma 1999). Because it focused specifically on the growth mindset message and offered strategies to do better by reducing anxiety, we thought that students would attend specifically to this more concise and focused message. Third, we hypothesized that students who watched the video would show interest and engagement in learning mathematics, at least as it relates to fractals.

\footnotetext{
${ }^{4}$ These ten teachers represented some $20 \%$ of the participating teachers from these four schools and almost $3 \%$ of the total number of teachers who participated fully in the larger research project.
} 
Table 2 Demographic information about the four schools

\begin{tabular}{lll}
\hline & Total number of students & Free and reduced lunch $(\%)$ \\
\hline Elementary School 1 & 803 & 41 \\
Elementary School 2 & 1,050 & 11 \\
Middle School 1 & 1,446 & 35 \\
Middle School 2 & 1,473 & 23 \\
\hline
\end{tabular}

For the second research question about teachers' perceptions of their students' interests in and confidence in succeeding in mathematics, we hypothesized that teachers' perceptions would mirror what students reported: that the game would focus on self-efficacy, that Brainology would focus on implicit theory of ability and the video would focus on interest in and enjoyment of learning mathematics.

\section{Coding the Student and Teacher Interviews}

We analyzed the student and teacher data separately. For the student data, we used three rounds of analysis: (1) creating code sets through writing analytical memos; (2) performing inter-coder agreement checks; and (3) engaging in research-team discussions to generate accounts of what we noticed (Corbin and Strauss 2008). First, two members of our research team listened to all 31 audio files and wrote analytical memos about the themes they noticed. They used these memos to generate an initial code set of 100 categories. Second, these two researchers chose four interviews they felt were emblematic of the rich themes they noticed throughout all the interviews to create a theoretical sample of the data set. Theoretical sampling allows researchers to formulate initial accounts while analyzing a subset of the data and then to analyze larger amounts of the data continually to explore such accounts further (Corbin and Strauss 2008; Creswell 2009). Our theoretical sample represented $12.5 \%$ of the student data.

Table 3 Demographic information about the ten teachers

\begin{tabular}{llllll}
\hline Grade & Digital resource & Gender & Ethnicity & Years teaching & Advanced degree? \\
\hline 5 & Game & F & White/Caucasian & $0-2$ & Masters \\
8 & Game & M & Asian/Pacific Islander & $6-10$ & \\
5 & Brainology & F & White/Caucasian & $6-10$ & \\
5 & Brainology & F & White/Caucasian & More than 10 & Masters \\
5 & Brainology & F & White/Caucasian & More than 10 & Masters \\
8 & Brainology & M & White/Caucasian & $0-2$ & \\
5 & Video & F & White/Caucasian & $0-2$ & \\
5 & Video & F & White/Caucasian & $2-6$ & Masters \\
5 & Video & F & White/Caucasian & More than 10 & \\
7 & Video & F & White/Caucasian & More than 10 & \\
\hline
\end{tabular}


Two additional research team members then listened to these four theoretical sample interviews to create their own analytical memos and code sets (Merriam 2009). Then, the four research team members were able to discuss codes and themes they had noticed within the same interviews making up the theoretical sample. Within both research teams, the team who initially analyzed all the interviews and the additional team who only analyzed the theoretical sample, one of the researchers was not part of the data collection and therefore analyzed the data uninfluenced by the themes of selfefficacy, implicit theories of ability, or interest and enjoyment. These updated codes were then used to re-analyze the full sample of student interviews, with an inter-rater reliability percentage of just over $80 \%$.

Third, the four research-team members came together to discuss the themes and codes they each noticed. This three-stage coding protocol helped us highlight what the students felt, reflected upon, or noted about in the digital resources. Through this process, the research team collapsed the 100 categories into 11 themes, which we then organized according to the three motivation categories of self-efficacy, implicit theories of ability, and interest and enjoyment.

For the teacher interviews, we used a two-stage expert analysis protocol (Corbin and Strauss 2008) to explore teacher reflection specific to motivating students using digital resources. Because the teachers were more forthright and direct in their interviews compared with the students, our analytical memos uncovered emergent themes organized by digital resource throughout all the teacher interviews relatively quickly. For the first part of the teacher data analysis, two of the research team members listened to all ten teacher interviews to create analytical memos. Then, all four research team members came together for several rounds of theoretical discussions to attempt to uncover emergent themes involving technology, sources of student motivation and the trains lesson, connecting these themes to what we found in the student data.

\section{Results}

Analyzing the student and teacher data revealed several themes relating technology and student self-efficacy, implicit theory, and interest. We start by reporting the main theme of motivation (as well as sub-themes) found amongst the student interviews. Ten of the thirty-two student interviews were about the game, fourteen interviews were about the Brainology modules, and eight interviews were about the video.

We then report on the main themes (technology, sources of student motivation, and the trains lesson) and sub-themes we found among the ten teacher interviews. Two of the ten interviews were about the game, four were about Brainology, and four were about the video. All ten mentioned the themes of technology, sources of student motivation, and the trains lesson.

\section{RQ1: Students' Perceptions of Motivational Constructs in the Digital Resources}

Our focus in this study related to how students perceived their motivation for learning mathematics. Here, we report students' views on the impact of the digital resource on their self-efficacy, implicit theory of ability, and their interest in and enjoyment of 
learning mathematics, as well as how they connected what they saw in the work they did during the 2-day trains problem exploration.

\section{Motivational Impact of the Technology for Learning Mathematics.}

We divided the motivational impact into three sub-themes: self-efficacy, implicit theories of ability, and interest and enjoyment. The first involved sources of selfefficacy, which fourteen of the thirty-two student interviews mentioned in one form or another. Given the sources that inform students' self-efficacy, we discuss this theme by considering the two most prominent sources that students mentioned: vicarious and mastery experiences. The former was the most common source of self-efficacy, being mentioned in ten of the overall interviews. Although we originally only designed the game to contain vicarious experiences, students' mentioned them in relation to all three digital resources - four of the ten game interviews, four of the fourteen Brainology interviews, and, surprisingly, two of the eight video interviews. (See Table 4.)

The vicarious experiences students acknowledged when playing the game were apparent because we designed the game to be an immersive experience in which the students 'became' the title character and also watched videos of real-life STEM professionals talking about struggling and then succeeding in mathematics. But we were surprised that students using the Brainology resource told us they felt they could relate to its characters and felt like they were in the animations with them. We were even more surprised that some students mentioned vicarious experiences in relation to the fractal video. For example, these two students identified with the narratives:

Interviewer: Would you say that watching the video made you feel more motivated to do well in math? [Question 3d in Appendix B]

Student 1: Yes, because it made me more think better, more, you know, like pay attention.

Table 4 Percentages of student interview responses to motivational constructs

\begin{tabular}{|c|c|c|c|c|c|}
\hline \multirow[t]{2}{*}{ Digital resource } & \multicolumn{2}{|l|}{ Self-efficacy } & \multirow[t]{2}{*}{ Implicit theory } & \multicolumn{2}{|c|}{ Interest and enjoyment $^{\mathrm{a}}$} \\
\hline & $\begin{array}{l}\text { Vicarious } \\
\text { experiences }\end{array}$ & $\begin{array}{l}\text { Mastery } \\
\text { experiences }\end{array}$ & & $\begin{array}{l}\text { Interesting/ } \\
\text { enjoyable }\end{array}$ & $\begin{array}{l}\text { Not interesting/ } \\
\text { enjoyable }\end{array}$ \\
\hline Game & $40 \%$ & $40 \%$ & & $50 \%$ & $20 \%$ \\
\hline Brainology & $29 \%$ & $7 \%$ & $36 \%$ & $71 \%$ & $57 \%$ \\
\hline Video & $25 \%$ & & & $50 \%$ & $50 \%$ \\
\hline Total $^{\mathrm{b}}$ & $31 \%$ & $16 \%$ & $16 \%$ & $59 \%$ & $44 \%$ \\
\hline
\end{tabular}

\footnotetext{
a The percentages for 'Interest and enjoyment' do not total to $100 \%$ because each interview was conducted in groups of 2 to 4 students, with some students within the same interview saying the resource was interesting and enjoyable while others said it was not.

${ }^{\mathrm{b}}$ The total of all students, regardless of the digital resource, who made reference to this motivational construct
} 
Student 2: It made me want to do, be a little bit smarter than that one [character in video].

Surprisingly, only five of the interviews mentioned mastery experiences despite our expecting students to attend to them in the game since it was built around mastery experiences. Four of these five were interviewed after playing the game, with students saying that the way the game introduced mathematics helped them see that it could be something they could succeed at: "Because if I can play a game and get all the [challenges] right, then I can take a test and I can do the same thing on a test". Another student remarked, "Like, if you just think of math as a game, math can be just as simple as the actual game". The one non-game interview that referred to mastery experiences was from Brainology®.

This small game percentage (four out the ten) was surprising, since we designed the game to be full of potential mastery experiences. Perhaps this was because the game's minimal guidance structure was too open-ended for students used to explicit instruction. In two of these interviews, the students mentioned a need for guidance. One student mentioned how initially none of them seemed to know what to do in the game. "It was really difficult because it didn't really give you that good of instructions to tell you what to do. It just gave you a pattern and blinks, so because you had nobody in the room, in the whole place even knew what to do, because it just gave us three colors, didn't tell us what to do with them."

The second motivation sub-theme was implicit theory of ability, with five interviews remarking on how the resource directly addressed the anxiety felt in mathematics class. Some students discussed mathematics learning in terms of the culture of mathematics in which it is couched, which seemed to trigger negative affective states for students. Fittingly, all five of these interviews were from the fourteen Brainology ${ }^{\circledR}$ interviews, which directly addressed how a fixed mindset can lead to mathematics anxiety. These students saw Brainology® as helping them get past this worry.

One fifth grade student commented, "I learned why I might be getting Ds and Cs, because I say bad things like, 'I'm going to fail the test and I hope I'll do good', but I need to change those bad things to good things, like 'I'm going to pass the test, I'm going to do good"'. A middle school student shared how Brainology® helped him deal with his academic anxiety, reporting, "I'm not doing a very good job in it, and I get all wound up and at home. I have the hardest time with my homework, so I found that it helped a lot, in how [to use] the breathing process and how to do things [that] work". While these students' reflections are not specific to mathematics learning, more than a third of the students working with the Brainology® resource were able to point to a fixed theory of ability to articulate their academic struggles.

The third motivation sub-theme involved interest and enjoyment. Nineteen interviews referred to how interesting or engaging the digital resource was. Five were from the game interviews, ten were related to Brainology®, and four from the video interviews. Students found the game enjoyable mainly because it was a video game. One student said, "I got kind of upset when we had to end the game, because I was doing so well and everything was just starting to make sense". This may suggest that students' interest in the game was directly tied to how well they thought they were performing - increased self-efficacy may have led to increased interest. Students 
working with the Brainology® resource seemed to enjoy it for two separate reasons. Some liked it because it allowed them to go online and surf the Internet. "It was an interesting day. I think [the teacher] was kind of irritated because everyone was, like, everyone wanted to go on a different website." In other words, students found the experience of being able to use the internet freely (in perhaps an unintended manner) to be enjoyable. Therefore, for some, the Brainology ${ }^{\circledR}$ resource was not necessarily interesting in and of itself.

Other students found Brainology® itself to be interesting. "I didn't really want to stop looking at the screen, like every time [the teacher] would say something I kind of ignored him just because I was interested in what we were watching, so I would keep watching it. That's how I know I was interested." Those students who found the video interesting pointed to the content of the fractals movie: "It was really interesting the way that you can figure out how far out you can go with the same shape going on, but smaller on the same one, smaller and smaller, until it could go to the size of an atom". Therefore, the video seemed successful at making the actual academic content interesting in and of itself.

On the other hand, fourteen of the interviews (almost half) commented on how the resource was neither interesting nor enjoyable. Two of these interviews were from the game interviews, eight involved Brainology®, and four discussed the video. For the game, students found that playing it was not challenging. "Sometimes it was just a little too easy because sometimes you just had to click and if it was the wrong one you just had to find the right one." For Brainology®, students found the lessons to be noninteractive, mentioning how the characters just "talked at them" or how the questions being asked were then quickly answered. One student commented, "Brainology®, it was kind of boring, but then it got started talking about, and it started showing pictures, I thought it was boring, but then it started showing questions, that was the ultimate boring". For the video, some students found it un-engaging when the subject matter was confusing or too complex. Others talked about how the video was informative, but not interactive enough to be engaging, one commenting, "It was interesting, but it didn't motivate me. They were informing you about it, you don't want to go and study the whole thing, you are just entertained at the moment".

Finally, we noticed that students complained or praised the aesthetics of the resource itself, even though these were not part of our interview questions. For instance, when speaking about the game, nearly a third of the students compared the game unfavorably with commercial Xbox or PlayStation games, critiquing the quality of the graphics, the interactivity of the game, the responsiveness of the interface, and the user interface design. One middle-school student complained about the slow control responses. "We kept bumping into walls and stuff, I'm like wait a minute, I don't like, this is not, like, chill." Additionally, when students commented on Brainology®, a quarter of them criticized the primitive animations. One interview unfavorably compared Brainology® to BrainPOP ${ }^{5}{ }^{5}$ a commercial educational website (www.brainpop.com) that students found much more "fun". By contrast, the video, which was commercially produced by PBS Nova, seemed to enthrall students, with half of the student interviews mentioning how much they liked it. One student enthusiastically remarked, "Oh my gosh it's so cool. Fractals are so cool! They're fun to watch and they're spiraling and all pretty!"

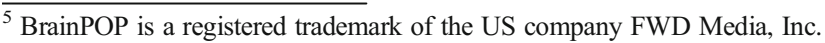


So even with a relatively minor exposure, students compare educational technology to prior experiences with commercial media. Perhaps if students' psychological needs (i.e., autonomy, competence and relatedness) are not satisfied, nor tied directly to the learning goals of the task or challenge, then students attend to the superficial qualities of the technology such as the graphics (see Przybylski et al. 2010).

\section{RQ2: Teachers' Perceptions of Students' Self-Efficacy, Implicit Theories of Ability, and Interests and Enjoyment in Mathematics Learning}

To add clarity to the themes revealed in the student interviews, we also interviewed these students' ten teachers. Of these ten teachers, two worked with the game, and four each with Brainology or the video.

First, both teachers working with the game expressed surprise at the way their students interacted with the game. For instance, in relation to who was doing well in the game and who struggled, one eighth-grade teacher noted: "I got to see some of them I wouldn't expect and they're all the way down here, you know, farther along in the game. Then I see some of them, they're just like stuck on the first one." This teacher also noticed that students, particularly students who normally struggled in math, seemed very motivated to beat the game, even getting angry when the class period was over. "They were doing their own thing. I mean, they got mad at me when I tried to stop at half an hour, because they really wanted to beat the game." This statement seems to corroborate the student-reported statement that beating the game (i.e., demonstrating the ability to accomplish a goal) seemed to fuel students' interest and enjoyment.

Second, all four of the Brainology® teachers mentioned how their students struggled to engage with the content or connect it to learning mathematics. One eighth-grade teacher mentioned that most of his students were just clicking through the slides. "The students thought it was corny, but funny [...] some of them were engaged, if they felt the content was interesting. Some of them were just clicking to get through it." A fifth-grade teacher observed, "most students didn't have a lot to say about it. It was hard to make any links or connections to the class lessons. But they retained the information."

Finally, in relation to the video, only two of the four teachers mentioned ways their students interacted with the fractals and narratives in the video. For instance, a fifth-grade teacher mentioned that, while the content might have been "over my students' heads", she claimed that, "most kids really enjoyed it, even though some of it was more conceptually difficult than typical classroom content. They understood some of it and didn't understand other parts, but they enjoyed it." These extracts from the teacher interviews indicate that selfefficacy in beating the game and enjoyment and interest in the video were the only motivational constructs from our framework that teachers seemed to notice in their students.

Overall, in regard to students, we found that most of them spoke about interest to learn mathematics in terms of self-efficacy (mainly by means of vicarious experiences and less in regard to mastery experiences). In fact, vicarious experiences based upon relating to the struggles of others in mathematics seemed very 
strong, with students highlighting these relationships throughout their work with all three digital resources. In comparison, perhaps unsurprisingly, only students using Brainology ${ }^{\circledR}$ spoke about implicit theory of ability.

\section{Discussion}

These results frame a snapshot of what students and teachers thought in terms of motivation, technology, and mathematics learning within a four-day-long mathematics curriculum. Because of the small data set and short intervention, we can neither generalize these results for all students and teachers nor show how students' motivation for learning mathematics grows over time. However, we can expand upon two conclusions with implications on how to build motivationally sound technology for mathematics learning. In our research design, students only engaged with the technology for one or two days at the time of the interviews, meaning that our data represent a snapshot reaction when encountering it for the first time. What can we conclude from this brief exposure?

First, we offer a few potential reasons for why students might have connected to the vicarious experiences available in Brainology® and the video. As Bandura (1997) noted, vicarious experiences become more effective when role models are perceived as highly similar to the observer. The stories were perhaps more accessible to the students because they were offered from a perspective of students struggling in school in a narrative format, a cultural form of storytelling to which students are accustomed (Bruner 1996), as opposed to what was present in the game, namely an interview with an adult from which students had to make meaning. When it comes to vicarious experiences, students do not engage when just hearing people talk about how they use math, but rather must be pulled into a narrative structure to which they can relate.

Because mathematics is often seen as a difficult subject, perhaps one salient factor that students use to identify vicarious models is the authenticity of a person's struggles with mathematics and how well they can relate to that struggle. A narrative structure might facilitate students' identification with a figure, if the narrative is one to which students can relate. In addition, students even create stories of how non-human objects move over time, anthropomorphizing mathematical objects in order to create vicarious experiences (Sinclair et al. 2009), showing the power of narrative structures in relation to how students relate to mathematics. As Nel Noddings (1991) observed, "stories have the power to direct and change our lives" (p. 157). River City, one of the successful learning technology interventions mentioned earlier, also contained a significant narrative component (Ketelhut et al. 2010).

Second, students generally noted the motivational constructs we expected them to notice. For the game, both vicarious and mastery experiences proved salient for $40 \%$ of the students, according to their reports. For those who participated in Brainology®, implicit theory of ability appeared salient for just over $35 \%$ of them. For the video, interest and enjoyment was salient for half of the group. We find these percentages relatively high considering the brief time. In sum, even with a short exposure, the motivational constructs we focused on proved salient for a fair number of the students.

Third, vicarious experiences were salient for some students across all three resources, and for more than a third of them in all. As a source of self-efficacy, such 
experiences seemed to resonate with students much more than mastery experiences, implicit theory of ability, or interest and enjoyment within a short time frame, suggesting students are attentive to vicarious experiences within these novel technology experiences, even over such a short time span. This supports Bandura's (1997) hypothesis that, in situations in which students have very few mastery experiences (here, in a digital environment in which they have not participated before), students' efforts to judge their capability to accomplish particular goals may need to come from observing similar experiences of others.

Finally, different resources seemed to target interest through two different pathways. In one, exemplified by the game, the resource presented incrementally challenging goals to students and made them aware of having accomplished them. Therefore, interest was an effect of students' perceptions of being successful in the endeavor. This aligns well with what Bandura (1997) has argued: "People display enduring interest in activities at which they feel efficacious and from which they derive self-satisfaction" (p. 219).

A second path to target interest arose from triggering a situational interest directly (Hidi and Renninger 2006). The video seemed to accomplish this for the few students who found fractals especially enjoyable. Unlike through selfefficacy, the direct method provides a less robust way to develop interest, and likely only sparks situational interest if the student finds that particular content surprising, personally relevant, or especially enjoyable. If students are to develop an interest in a task or a field of study, not only do they need to be drawn toward it, but they also need to know that they can successfully attain desired goals (Bandura 1997).

Bandura has also argued (a) that there might be a temporal lag between when self-efficacy first increases and when interest subsequently develops and (b) that there may be a threshold pattern such that when students reach a moderate level of self-efficacy any further gains do not result in any further gains in interest. These two phenomena - the temporal lag and the threshold are not mutually exclusive and, in fact, might often be working in tandem.

This could certainly be the case in our present study. If students were able to reach a moderate level of self-efficacy by succeeding in the game, they may then have begun to experience increases in their interest as they gained confidence in being able to succeed at harder mathematical challenges. This is speculative, however, and further research could explore whether these two phenomena linking interest and self-efficacy development emerge.

\section{Implications}

What do these results mean? The literature continually points to how the implementation of technology itself is important - knowing the theory or the science behind motivation is one dimension, but connecting those frameworks to concrete situations and people points to the importance of translational research (see Bandura 2004). The resources were tightly connected and targeted, and our implementation was carefully monitored and administrated. Additionally, even with only a brief exposure, we were able to see that the constructs we intended to target proved somewhat salient to students. 
What are some implications for instructional designers and educational technologists? Social cognitive theory (Bandura 1986) suggests that the effectiveness of a vicarious experience hinges on the relatability of the character to the intended participant. However, despite our best efforts to produce a resource (the game) that reflected this construct of vicarious experiences, students seemed to respond to the vicarious experiences in all three of the resources.

We conclude by calling for more research in exploring the motivational affordances of technology, specifically how students hear and translate motivational messages present in the technology. Perhaps, with prolonged exposure, we might be able to see exactly how students internalize specific motivational constructs. We have seen exciting results over a very short time-span, but because we did not collect data regarding this question of translational research (i.e. how students internalized these motivational constructs as a consequence of our implementation) we are left wondering how students did so.

Our short time frame reflects the challenges of doing classroom-based research in today's accountability-based educational system. It also meant that we were unable to collect data about changes in students' mathematical content knowledge before and after the technology interventions, meaning that we were unable to connect the data on motivation to learn mathematics with how the students were actually learning mathematics. We are left wondering what impact on actual mathematics learning these digital resources had.

We also note that the offered tasks were limited to what could be accomplished in a short time frame, meaning that we could not build our intervention around robust technology that requires appreciably more time for students to learn how to use them (e.g. dynamic geometry software, statistical modeling applications). We would like to continue this vein of research and explore connections between motivation constructs and larger educational technologies that capitalize on the specific aspects of the classroom. For example, our students mentioned how they liked playing the game in class because they got help from their peers and their teacher, showcasing an advantage that classroom-based technology has over commercial video games: classroom camaraderie and shared experiences. We hope our work adds to the growing literature exploring connections between educative mathematics technology and motivational constructs.

In this study, we saw a glimmer of hope in how even a brief encounter led to students recognizing and responding to the motivational constructs and noticing vicarious experiences everywhere. Our effectiveness as designers of educational technology hinges on our ability to understand not only how to embed motivational constructs within the technology, but also how students and teachers translate these constructs into designs and practices that align with how students actually use and experience them.

Acknowledgment This work was supported by the National Science Foundation under Grant DRL 0929575 (Dede and Star). We thank Stephanie Fitzgerald, Kinga Petrovai, Bharat Battu, Lauren Schiller, Chad Desharnais, and Amy Venditta for all their help in collecting and analyzing this data, and Megan Taylor for guidance in the analysis. And a very special thanks to all the teachers and students working with us on the TESLA project. 


\section{Appendix A}

TESLA Trains Lesson worksheet

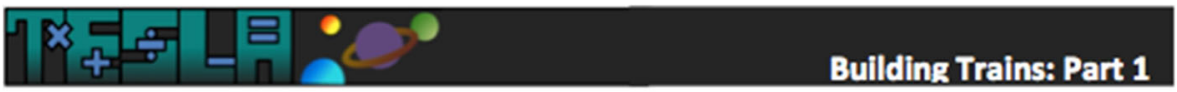

\section{MISSION: To determine how many trains of length 7 can be built.}

\section{Sub-Mission \#1:}

To determine how many different trains of length 4 can be built.

Here are two examples:
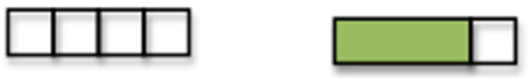

\section{Tasks:}

(a.) Make a list of all the length 4 trains that can be built.

(b.) Compare your list with a friend to make sure you both found all possible trains.

(c.) Talk with your friend about how you know you found every possible train.

(d.) Put your list(s) on a poster to share.

\section{Appendix B}

\section{Student Interview Protocol}

Background

1. Could you tell me a little about the types of technology that you usually use in your math class this year? For example, computers, calculators, or other electronic things.

a What do you usually use these technologies to do?

b How do you feel about using technology in your math class (is it useful, interesting? Does it help you learn math better?)

2. Could you describe how motivated and confident you are in doing math?

a What motivates you to do well and keep trying in your math class even if it's really hard?

b If you're given a tough math problem, how likely are you to try really hard until you get it? (Explain why you are or are not likely to try hard until you get it). 
c How useful to you is the kind of math that you're learning in class this year? How useful will it be for your future?

Views on the intervention

3. Could you tell me about the technology activity that you participated in during the first day.

a Was it interesting? Was it fun? In what ways?

b Do you think that the technology activity had anything to do with math or with what you were learning in math class?

c Did the technology activity make you see math in a different way? Science and math careers in a different way?

d Did the technology activity make you feel more motivated to do well in math? (If yes, how so? If no, why not?)

4. Compared to what you normally do in your mathematics classes, was the technology activity more or less fun? Explain your reasons.

\section{Appendix C}

\section{Teacher Interview Protocol}

\section{Background}

1. Could you tell me a little about how you use technology in your math classroom (such as computers, calculators, smart boards)?

a How do you normally use these technologies? Can you give me a specific example of when you used technology in your math class recently?

b How do your students normally respond to the technology that you use?

2. Could you give me a picture of a normal week for one of your math classes?

a What is a typical lesson's flow? How does it begin? What happens next? How does it end?

b How do you use the textbook in planning?

c How do you use the textbook during class?

d What is your typical role in class?

e How are the students typically engaged in mathematics (e.g., small group work, note-taking)?

3. Could you describe the range of motivation and confidence levels of your students?

a What motivates your students to engage in the mathematics?

b What do you think is the best way to build your students' confidence in math? 
c When you give your students a really tough problem, can you describe why some students give up early and why some kids persevere?

Views on the technology

4. Can you tell me what your students thought about the technology activity that they did? Were they engaged? Did they like it? Why did or didn't they like it?

5. Did you think that the technology activity was something that benefitted the students? Why or why not?

6. Do you think that you would want to do something like this technology activity again? Explain.

Views on the mathematics lessons

7. Could you tell me about how your students responded to the math lessons?

a. Were they interested? Engaged? In what ways?

b. Was the mathematics too challenging or too easy?

8. What mathematics did the students learn? What learning goals were met?

What evidence of this learning did you glean?

9. How did the changes you made to the lesson materials affect the students' engagement and learning?

10. How did the changes you made to the implementation of the lesson materials affect the students' engagement and learning?

11. If you could teach these lessons again, what would you do differently? Why?

\section{References}

Adelman, C. (2006). The toolbox revisited: paths to degree completion from high school through college. Washington: U.S. Department of Education.

Archambault, I., Eccles, J., \& Vida, M. (2010). Ability self-concepts and subjective value in literacy: joint trajectories from grades 1 through 12. Journal of Educational Psychology, 102(4), 804-816.

Ashcraft, M., \& Krause, J. (2007). Working memory, math performance, and math anxiety. Psychonomic Bulletin \& Review, 14(2), 243-248.

Bandura, A. (1986). Social foundations of thought and action: a social cognitive theory. Englewood Cliffs: Prentice-Hall.

Bandura, A. (1997). Self-efficacy: the exercise of control. New York: W. H. Freeman.

Bandura, A. (2004). Social cognitive theory for personal and social change by enabling media. In A. Singhal, M. Cody, E. Rogers, \& M. Sabido (Eds.), Entertainment-education and social change: history, research, and practice (pp. 75-96). Mahwah: Lawrence Erlbaum.

Barab, S., Dodge, T., Jackson, C., \& Arici, A. (2003). Technical report on quest Atlantis (Vol. 1). Bloomington: Indiana University Center for Research on Learning and Technology.

Blackwell, L., Trzesniewski, K., \& Dweck, C. (2007). Implicit theories of intelligence predict achievement across an adolescent transition: a longitudinal study and an intervention. Child Development, 78(1), 246-263.

Brown, S., \& Lent, R. (2006). Preparing adolescents to make career decisions. In F. Pajares \& T. Urdan (Eds.), Adolescence and education self-efficacy beliefs of adolescents (Vol. 5, pp. 201-223). Greenwich: Information Age.

Bruner, J. (1996). The culture of education. Cambridge: Harvard University Press.

Chen, J., Zap, N., \& Dede, C. (2012). Using virtual environments to motivate students to pursue STEM careers: an expectancy-value model. In S. D'Agustino (Ed.), Immersive environments, augmented realities, and virtual worlds: assessing future trends in education (pp. 42-56). Hershey: IGI Global. 
Chen, J., Metcalf, S., \& Tutwiler, S. (2014). Motivation and beliefs about the nature of scientific knowledge within an immersive virtual ecosystems environment. Contemporary Educational Psychology, 39(2), $112-123$.

Chen, J., Tutwiler, S., Metcalf, S., Kamarainen, A., Grotzer, T., \& Dede, C. (2016). A multi-user virtual environment to support students' self-efficacy and interest in science: a latent growth model analysis. Learning and Instruction, 41, 11-22.

Corbin, J., \& Strauss, A. (2008). Basics of qualitative research: techniques and procedures for developing grounded theory (3rd ed.). Thousand Oaks: Sage.

Creswell, J. (2009). Research design: qualitative, quantitative, and mixed methods approaches (3rd ed.). Thousand Oaks: Sage.

Cury, F., Elliot, A., Da Fonseca, D., \& Moller, A. (2006). The social-cognitive model of achievement motivation and the $2 \times 2$ achievement goal framework. Journal of Personality and Social Psychology, 90(4), 666-679.

Donohoe, C., Topping, K., \& Hannah, E. (2012). The impact of an online intervention (Brainology) on the mindset and resiliency of secondary school pupils: a preliminary mixed methods study. Educational Psychology, 32(5), 641-655.

Dweck, C. (2000). Self-theories: their role in motivation, personality, and development. Philadelphia: Psychology Press.

Dweck, C. (2007). Is math a gift? beliefs that put females at risk. In S. Ceci \& W. Williams (Eds.), Why aren't more women in science? Top researchers debate the evidence (pp. 47-55). Washington: American Psychological Association.

Dweck, C., \& Leggett, E. (1988). A social-cognitive approach to motivation and personality. Psychological Review, 95(2), 256-273.

Dweck, C., \& Master, A. (2009). Self-theories and motivation: Students' beliefs about intelligence. In K. Wentzel \& A. Wigfield (Eds.), Handbook of motivation at school (pp. 123-140). New York: Routledge.

Eccles-Parsons, J., Adler, T., Futterman, R., Goff, S., Kaczala, C., Meece, J., \& Midgley, C. (1983). Expectancies, values, and academic behaviors. In J. Spence (Ed.), Achievement and achievement motivation (pp. 75-146). San Francisco: W. H. Freeman.

Good, C., Rattan, A., \& Dweck, C. (2012). Why do women opt out? sense of belonging and women's representation in mathematics. Journal of Personality and Social Psychology, 102(4), 700-717.

Grant, H., \& Dweck, C. (2003). Clarifying achievement goals and their impact. Journal of Personality and Social Psychology, 85(3), 541-553.

Hidi, S., \& Renninger, K. (2006). The four-phase model of interest development. Educational Psychologist, 41(2), 111-127.

Hoffer, T., Venkataraman, L., Hedberg, E., \& Shagle, S. (2007). Final report on the national survey of algebra teachers for the national math panel. Chicago: National Opinion Research Center at the University of Chicago (http://www2.ed.gov/about/bdscomm/list/mathpanel/final-report-algebra-teachers.pdf).

Ketelhut, D., Nelson, B., Clarke, J., \& Dede, C. (2010). A multi-user virtual environment for building and assessing higher order inquiry skills in science. British Journal of Educational Technology, 41(1), 56-68.

Liu, M., Hsieh, P., Cho, Y., \& Schallert, D. (2006). Middle school students' self-efficacy, attitudes, and achievement in a computer-enhanced problem-based learning environment. Journal of Interactive Learning Research, 17(3), 225-242.

Ma, X. (1999). A meta-analysis of the relationship between anxiety toward mathematics and achievement in mathematics. Journal for Research in Mathematics Education, 30(5), 520-540.

Merriam, S. (2009). Qualitative research: a guide to design and implementation (2nd ed.). San Francisco: Jossey-Bass.

Moos, D., \& Marroquin, E. (2010). Multimedia, hypermedia, and hypertext: motivation considered and reconsidered. Computers in Human Behavior, 26(3), 265-276.

Moses, R., \& Cobb, C. (2001). Radical equations: math literacy and civil rights. Boston: Beacon Press.

Noddings, N. (1991). Stories in dialogue: caring and interpersonal reasoning. In C. Witherell \& N. Noddings (Eds.), Stories lives tell: narrative and dialogue in education (pp. 157-170). New York: Teachers College Press.

NRC. (2001). Adding it up: helping children learn mathematics. Washington: National Research Council.

Pajares, F., \& Urdan, T. (2006). Adolescence and education: Vol. 5 self-efficacy beliefs of adolescents. Greenwich: Information Age.

Przybylski, A., Rigby, S., \& Ryan, R. (2010). A motivational model of video game engagement. Review of General Psychology, 14(2), 154-166. 
Rattan, A., Good, C., \& Dweck, C. (2012). "It's OK - not everyone can be good at math": instructors with an entity theory comfort (and demotivate) students. Journal of Experimental Social Psychology, 48(3), 731737.

Schwarz, M., \& Jersey, B. (2009). Fractals: hunting the hidden dimension (NOVA). Arlington: Public Broadcasting Service (http://www.pbs.org/wgbh/nova/physics/hunting-hidden-dimension.html).

Sinclair, N., Healy, L., \& Sales, C. (2009). Time for telling stories: narrative thinking with dynamic geometry. ZDM: The International Journal on Mathematics Education, 41(4), 441-452.

Star, J., Chen, J., Taylor, M., Durkin, K., Dede, C., \& Chao, T. (2014). Studying technology-based strategies for enhancing motivation in mathematics. International Journal of STEM Education, 1, 7.

Stein, M., \& Lane, S. (1996). Instructional tasks and the development of student capacity to think and reason: an analysis of the relationship between teaching and learning in a reform mathematics project. Educational Research and Evaluation, 2(1), 50-80. 